\title{
COMPLICATION AFTER MINIMAL INVASIVE REPAIR OF PECTUS EXCAVATUM
}

\author{
Vlad Laurentiu David ${ }^{1,2}$, Marius Calin Popoiu ${ }^{1,2}$, Corina Maria Stanciulescu ${ }^{1,2}$, Eugen Sorin \\ Boia $^{1,2}$
}

Abstract

Background: Minimal invasive repair of Pectus Excavatum (MIRPE) has now become the main therapeutic method for Pectus Excavatum (PE) in children. This study focuses on our experience with MIRPE with special emphasis on the complication of the surgical procedure. Methods: Since 2007 we have performed 33 minimal invasive repairs of PE. The surgical technique was the standard MIRPE technique with the modifications we have previously described. Results: We had no intraoperative accidents and no fatalities. Postoperative complications occurred in 8 of the 33 cases. Most of the complications (4 cases) were wound-related, with dehiscence or infection. Postoperative pleural effusions occurred in 3 patients, pneumothorax in 1 case and pericarditis in 1 case. All the complications occurred within 4 weeks from the surgical intervention. Reoperations were necessary in 2 cases, all of them for closing the dehiscent surgical wound. We had no bar shifts. The bar was removed in 20 patients after a period ranging from 1 to 3 years. Conclusion: Our results are comparable with those of the early reports of this technique and are subject to continuous improvement.

Keywords: Pectus Excavatum, minimal invasive repair, surgical treatment, complications, outcome, healthcare quality

\section{Introduction}

More than two decades after it was first performed and one decade after the first reports about the procedure were published, minimal invasive repair of Pectus Excavatum (MIRPE) has now become the main therapeutic method for Pectus Excavatum (PE) in children (1). Even though there is no consensus regarding the origin of the disease, there is consensus over the treatment of the disease and the benefits of the minimal invasive approach: less trauma to the tissue, and tiny, almost negligible, postoperative scarring on the skin. Several studies have concluded that there are real benefits for the patients that underwent repair of the chest deformity, in terms of cardio-vascular function, release of the symptoms, esthetics and body image $(2,3,4,5,6)$.
Various improvements and modification of the technique, as well as reports of the results, are published every year all over the world. Along with the learning curve and improvement of the technique complication rates have decreased gradually: from $12 \%$ in the first decade to $1 \%$ in the second decade $(4,7)$. Severe complications like bare shift, heart trauma, pleurisy or pericarditis occur now in less than $1 \%$ of the patients (4).

In 2007 we were the first surgical team composed exclusively of Romanian doctors to perform this type of surgical intervention, and we reported our preliminary results in 2010 (6). Since then MIRPE has become the standard surgical procedure in our departments. A comprehensive analysis of our experience with both opened and minimal invasive repair of $\mathrm{PE}$ in children reveals, without doubt, the superiority of the latter in both safety and outcomes of the treatment (9).

This study focuses on our experience with MIRPE with special emphasis on the complication of the surgical procedure.

\section{Material and method}

Since 2007 we have performed 33 minimal invasive repairs of PE. Our patients were 6 girls and 27 boys. The age of the patients ranged between 8 and 20 years, with a median of 15.2 years. One patient was less than 12 years old ( 8 years) at the time of the operation. The deformity was symmetric in $17(5 \%)$ patients. Symptoms were present in 21 patients $(63.6 \%)$, effort dyspnea in 20 cases and recurrent chest pain in 2 patients. In 2 cases $(10 \%)$ palpitations were associated to effort dyspnea. Clinical exam revealed different degrees of alteration of the cardiac function of in 18 patients $(54.5 \%)$. In $5(15 \%)$ patients Marfan syndrome was also present. In one case, a 19 years old female, the deformity reoccurred after a previous Ravitch correction of PE at the age of 6 . The surgical technique was the standard MIRPE technique with the modifications we have previously described, Nicodin et al. $2010(8)$

${ }^{1}$ Department of Pediatric Surgery and Orthopedics "Victor Babes" University of Medicine and Pharmacy Timisoara, Romania

${ }^{2}$ Department of Pediatric Surgery and Orthopedics “Louis Turcanu” Emergency Children's Hospital Timisoara, Romania

E-mail: david.vlad@umft.ro, mcpopoiu@yahoo.com, stanciulescucorina@yahoo.com, boiaeugen@yahoo.com 
Table 1. Postoperative complications

\begin{tabular}{l|l} 
Complications & No. of patients \\
Wound dehiscence/ infections & 4 \\
Pleural effusions & 3 \\
Pneumothorax & 1 \\
Pericarditis & 1 \\
Bar shift & 0 \\
Reoperation & 5 \\
Overall complications & 8 \\
\hline
\end{tabular}
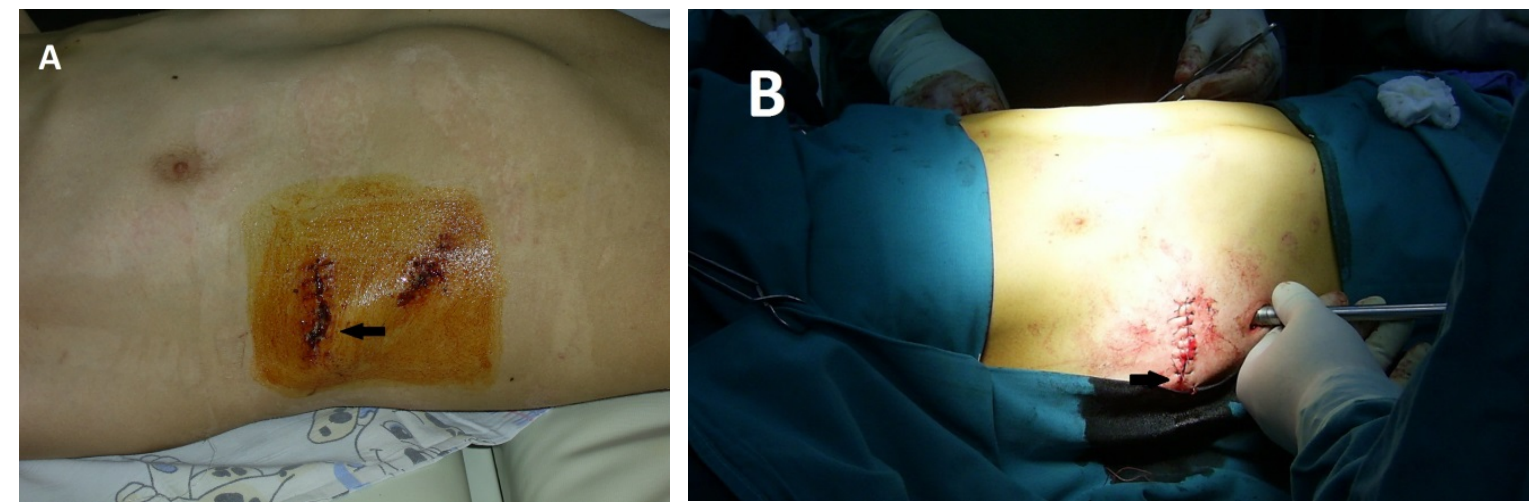

Fig. 1. A. Partial dehiscence of the surgical wound, 14 days after surgery in a 14 years old boy with Marfan syndrome; note the elevation of the skin produced by the insufficient bending of the end of the bar. B. Same patient at the end of the surgical intervention

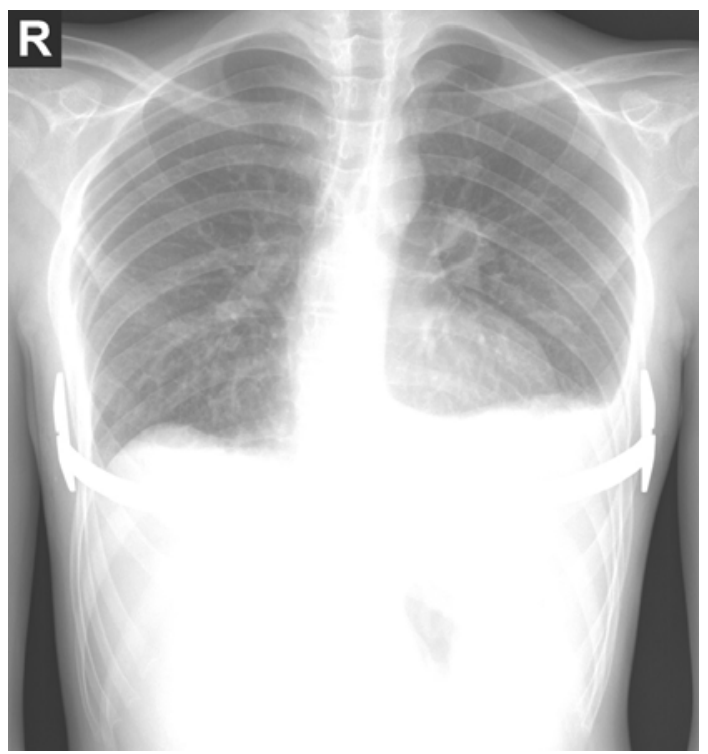

Fig. 2. Postoperative pleural effusion 14 days from surgery

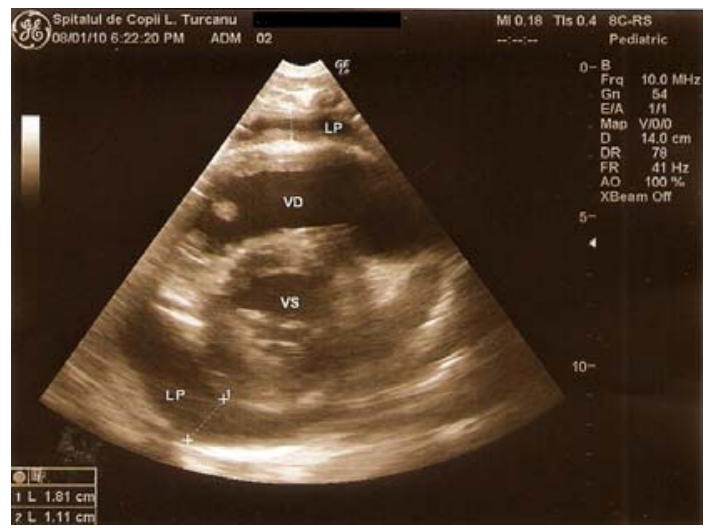

Fig. 3. Image of the cardiac ultrasound of 15 years old boy with pericarditis 


\section{Results}

The operative time ranged from 60 to 120 minutes with a median of 71.5 minutes. Hospital stay ranged from 6 to 40 days, mean 12 days. We had no intraoperative accidents. We used postoperative pleural drain in 26 of the cases, 25 unilateral and one bilateral. The pleural drain was kept in place for a mean of 3.69 days (1-14 days). Postoperative complications occurred in 8 of the 33 cases $(24.8 \%)$ (Table 1). We had no fatalities. Most of the complications (4 cases) were wound-related, with dehiscence or infection. Wound dehiscence was the most prevalent complication in our series, and our observation was that it was related to an insufficient bending of the tip of the Lorenz bar. In the surgical technique we use, we prefer to leave the end of the bar and the lateral stabilizer in the subcutaneous tissue (not sub-muscular) (Fig. 1). Postoperative pleural effusions occurred in 3 patients, in 2 of them spontaneous resolution after conservative treatment occurred and in one case needle aspiration was performed (Fig. 2). In one patient pneumothorax occurred 24 hours after surgery and pleural drainage was necessary.

Pericarditis occurred in one of the cases. It was the most severe complication that we were forced to face (Fig. $3)$. The complication occurred during the first week after surgery. We performed a pericardial puncture, and the aspirated liquid was cultured. The patient received both antibiotic treatment and prolonged anti-inflammatory medications. The pericarditis was slowly relieved, but the patient required prolonged hospitalization (40 days).

All the complications occurred within 4 weeks from the surgical intervention. Reoperations were necessary in 5 cases, 4 for closing the dehiscent surgical wound and 1 pneumothorax drainage. The bar was removed in 20 patients after a period ranging from 1 to 3 years. We had no complications after bar removal.

\section{Discussions}

First reports after the introduction of MIRPE in 1998 by Nuss indicated a higher rate of complications for the minimal invasive technique (6). After the early learning experience, complication rate dropped significantly but still lays around $15 \%(4,10,11,12)$. The overall complication rate of $24 \%$ in our series is still high compared to most other current reports, but it may be compared to the early results of other series reported in literature, even by those who implemented this technique (1).

Wound infections occur in up to $1,5 \%$ of the patients and is a major cause of surgical reintervention (13). In our series wound dehiscence and infection was the most prevalent complication. In 4 of these patients we were forced to do a revision of the surgical wound. A direct observation was that in these patients the bar was insufficiently bent with the tip of the bar, resulting in pressure on the wound sutures. In all three cases debridement of the necrotic tissue and secondary closure of the wound was performed. In the following cases we tried to bend the bar a little more so that the tips of the bar would dig in the lateral thoracic wall.

Pleural complications are probably the most common complications after Nuss procedure. Postoperative pneumothorax occurs in up to $7 \%$ of the cases, while pleural effusion occurs as high as $3 \%(10,11,12,14,15$, 16). In our series pneumothorax occurred in 1 of the 33 cases but when analyzing this result we have to consider that in most of the cases (24 out of 33) we used postoperative pleural drainage for at least 24 hours. So 1 out of 7 non-drained cases means approximately $14 \%$. So. it is our belief that, because the patient is immobilized in bed at least for the first 24 hours, a 1 to 2 days passive drainage of the right pleural cavity will permit the evacuation of any residual intrapleural fluids. On the other hand, pleural effusions occurred in 3 cases, and in 1 of them we were forced to perform needle aspiration. All cases went well after treatment, the Pectus bar was kept in place, and nickel allergy may be excluded.

Cardiac or pericardial lesions are probably the most severe complications after MIRPE. Intraoperative accidental heart or pericardial perforation may lead to intraoperative death $(17,18,19)$. Fortunately, we have had no such fatal complications. One of the patients developed a pericardial effusion in the first days after surgery. Because nickel allergy couldn't be proved, the cause of the pericardial effusion was probably a pericardial trauma during surgery. The problem was resolved with conservative treatment, without sequelae. Nevertheless, this complication increased significantly the hospital stay up to 40 days and consequently the costs of treatment increased. Perhaps one of the most important features in our series is that we had no bar shift. Bar shift was initial one of the most important postoperative complication in Nuss technique. Initial reports indicated a rate up to $15 \%(12$, 20). The consequent use of the lateral stabilizers reduced this complication to less than $1 \%$ (4). We always use lateral stabilizers and suture them tight to the muscular and subcutaneous tissue, and so we were never forced to deal with this category of problem.

\section{Conclusions}

The advantages of minimal invasive repair of PE are clear. Nevertheless, together with benefits there are some pitfalls that may overshadow the remarkable results of the treatment. We had an overall complication rate of $24 \%$.

The most prevalent complication was wound related and the most severe was pericarditis. We had no fatalities and no bar shifts. Our results are comparable with those of the early reports of this technique and are subject to continuous improvement. 


\section{References}

1. Nuss D, Kelly RE jr, Croitoru DP, et al. A 10 years review of a minimal invasive technique for the correction of PE. J Pediatr Surg 1998;33:545-552

2. David VL, Izvernariu DA, Popoiu CM, Puiu M, Boia ES. Morphologic, morphometrical and histochemical proprieties of the costal cartilage in children with pectus excavatum. Rom J Morphol Embryol. 2011;52(2):6259.

3. David VL, Cerbu S, Haragus H, Popoiu MC, Stanciulescu CM, Cozma G, Burlacu O, Boia ES. Costal Cartilages Do Not Overgrow in Patients with Pectus Excavatum. Med Princ Pract. 2016;25(6):533538. Epub 2016 Aug 16.

4. Nuss D. Minimally invasive surgical repair of PE. Semin Pediatr Surg. 2008 Aug;17(3):209-17

5. Neviere R, Montaigne D, Benhamed L, Catto M, Edme JL, Matran R, Wurtz A. Cardiopulmonary response following surgical repair of $\mathrm{PE}$ in adult patients. Eur $\mathrm{J}$ Cardiothorac Surg. 2011 Aug;40(2):e77-82. doi:10.1016/j.ejcts.2011.03.045. Epub 2011 May 12.

6. Metzelder ML, Kuebler JF, Leonhardt J, Ure BM, Petersen C. Self and parental assessment after minimally invasive repair of PE: lasting satisfaction after bar removal. Ann Thorac Surg 2007;83: 1844-9.

7. Park HJ, Lee SY, Lee CS. Complications associated with the Nuss procedure: analysis of risk factors and suggested measures for prevention of complications. J Pediatr Surg. 2004 Mar;39(3):391-5; discussion 391-5

8. Nicodin A, Boia ES, Popoiu MC, Cozma G, Nicodin G, Badeti R, Trailescu M, Adam O, David VL. Preliminary results after Nuss procedure. Chirurgia (Bucur). 2010 Mar-Apr;105(2):203-10.

9. Boia ES, Popoiu MC, David VL et all. Comparative analysis of modified Ravitch and minimal invasive Nuss procedures for the correction of PE in children. Jurnalul Pediatrului - Year XIII, Vol. XIII, Nr. 49-50, January-June 2010; p 80-85

10. Fonkalsrud EW, Beanes S, Hebra A, Adamson W, Tagge E. Comparison of minimally invasive and modified Ravitch PE repair. J Pediatr Surg. 2002 Mar;37(3):413-7.

11. Ong CC, Choo K, Morreau P. The learning curve in learning the curve: a review of Nuss procedure in teenagers. ANZ J Surg 2005;75:421-4.

12. Croitoru DP, Kelly RE, Goretsky MJ, Lawson ML, Swoveland B, Nuss D. Experience and modification update for the minimally invasive Nuss technique for PE repair in 303 patients. J Pediatr Surg 2002;37: 43745.

13. Shin S, Goretsky MJ, Kelly RE Jr, Gustin T, Nuss D. Infectious complications after the Nuss repair in a series of 863 patients. J Pediatr Surg. 2007 Jan;42(1):87-92.

14. Nuss D. Review and discussion of the complications of minimally invasive PE repair. Eur J Pediatr Surg 2002;12:230-4

15. Van Renterghem KM, von Bismarck S, Bax NM, Fleer A, Höllwarth ME. Should an infected Nuss bar be removed? J Pediatr Surg. 2005 Apr;40(4):670-3.

16. Hebra A, Swoveland B, Egbert M, Tagge EP, Georgeson K, Othersen HB Jr et al. Outcome analysis of minimally invasive repair of PE: review of 251 cases. J Pediatr Surg 2000;35:252- 8.

17. Moss R, Albanese CT, Reynolds M. Major complications after minimally invasive repair of $\mathrm{PE}$ : case reports. J Pediatr Surg 2001;36:155-8.

18. Marusch F, Gastinger I.Life-threatening complication of the Nuss-procedure for funnel chest. A case report Zentralbl Chir. 2003 Nov;128(11):981-4.

19. Bouchard S, Hong AR, Gilchrist BF, Kuenzler KA. Catastrophic cardiac injuries encountered during the minimally invasive repair of PE. Semin Pediatr Surg. 2009 May;18(2):66-72. doi: 10.1053/j.sempedsurg.2009.02.002

20. Nuss D. Review and discussion of the complications of minimally invasive PE repair. Eur J Pediatr Surg $2002 ; 12: 230-4$ 